\title{
Cathepsin B is highly expressed in pancreatic cancer stem-like cells and is associated with patients' surgical outcomes
}

\author{
TAKUYA FUJIMOTO $^{1}$, RYOUICHI TSUNEDOMI ${ }^{1}$, SATOSHI MATSUKUMA ${ }^{1}$, KIYOSHI YOSHIMURA ${ }^{2}$, \\ ATSUNORI OGA $^{3}$, NOBUYUKI FUJIWARA ${ }^{1}$, YASUHIRO FUJIWARA ${ }^{1}$, HIROTO MATSUI ${ }^{1}$, \\ YOSHITARO SHINDO ${ }^{1}$, YUKIO TOKUMITSU ${ }^{1}$, NOBUAKI SUZUKI ${ }^{1}$, SHOGO KOBAYASHI $^{4}$, \\ SHOICHI HAZAMA ${ }^{5}$, HIDETOSHI EGUCHI ${ }^{4}$ and HIROAKI NAGANO ${ }^{1}$
}

\begin{abstract}
${ }^{1}$ Department of Gastroenterological, Breast and Endocrine Surgery, Yamaguchi University Graduate School of Medicine, Ube, Yamaguchi 755-8505; ${ }^{2}$ Department of Clinical Research in Tumor Immunology, Showa University Clinical Research Institute for Clinical Pharmacology and Therapeutics, Setagaya, Tokyo 157-8577; ${ }^{3}$ Department of Molecular Pathology, Yamaguchi University Graduate School of Medicine, Ube, Yamaguchi 755-8505; ${ }^{4}$ Department of Gastroenterological Surgery, Osaka University Graduate School of Medicine, Suita, Osaka 565-0871; ${ }^{5}$ Department of Translational Research and Developmental Therapeutics Against Cancer, Yamaguchi University School of Medicine, Ube, Yamaguchi 755-8505, Japan
\end{abstract}

Received February 28, 2020; Accepted September 15, 2020

DOI: $10.3892 / 01.2020 .12291$

\begin{abstract}
Cancer stem-like cells (CSLCs) in solid tumors are resistant to conventional chemotherapy and molecularly targeted therapy, which is thought to contribute to cancer recurrence and metastasis. The present study aimed to identify biomarkers for pancreatic CSLCs (P-CSLCs). Using our previously reported methods, P-CSLC-enriched populations were generated from pancreatic cancer cell lines. The protein expression profiles of these populations were compared with those of parental cells using two-dimensional electrophoresis, tandem mass spectrometry, flow cytometry and immunohistochemistry. Protein expression in surgical specimens was also evaluated for relationships with clinical outcomes. A lysosomal cysteine protease, cathepsin B (CTSB), was significantly upregulated in P-CSLCs compared with that in the parental cells, as shown using western blotting. Flow cytometry analysis also confirmed that CTSB was more highly expressed on the surface of P-CSLCs compared with that on parental cells. Moreover, PCLCs had elevated cellular secretions of CTSB compared with the parental cells. Finally,
\end{abstract}

Correspondence to: Dr Hiroaki Nagano, Department of Gastroenterological, Breast and Endocrine Surgery, Yamaguchi University Graduate School of Medicine, 1-1-1 Minami-Kogushi, Ube, Yamaguchi 755-8505, Japan

E-mail: hnagano@yamaguchi-u.ac.jp

Abbreviations: $\mathrm{CTSB}$, cathepsin $\mathrm{B}$; $\mathrm{CD} 44 \mathrm{v}$, variant isoforms of CD44; CALR, calreticulin; CSC, cancer stem cell; CSLC, cancer stem-like cell; P-CSLC, pancreatic cancer stem-like cell

Key words: pancreatic cancer, cancer stem cells, biomarker, cathepsin B
CTSB expression was evaluated in 69 resected tumor specimens, and high expression was associated with the patients' clinicopathological features and surgical outcomes. The present results suggested that CTSB is a biomarker for poor survival in patients with pancreatic cancer, which is possibly associated with P-CSLCs. This novel biomarker may also have potential as a therapeutic target.

\section{Introduction}

Pancreatic cancer is the fourth leading cause of cancer-associated death in Japan according to Japan's National Cancer Center (1). Most patients present with locally advanced disease or systemic metastasis at diagnosis, at which point only $15-20 \%$ of tumors are resectable (2). Furthermore, pancreatic cancer has a high relapse rate after radical surgery (relapse-free survival rate, 6.7-13.4 months; five-year survival rate, 10.4-20.7\%) and is often resistant to conventional chemotherapy and radiation therapy $(3,4)$. There is ongoing research regarding effective adjuvant chemotherapy and new immunotherapies (5), although new therapeutic targets are still needed. Based on the high rates of local recurrence and distant metastasis, the lack of response to conventional treatment may be associated with the presence of cancer stem-like cells (CSLCs) (6-9); however, further research is needed regarding the biological properties of CSLCs and how these may be therapeutically targeted.

The tumorigenic subpopulation of pancreatic cancer cells is reported to have high expression of CD44, CD24 and epithelial-specific antigen (10). Furthermore, pancreatic (P-)CSLCs are characterized by increased expression of aldehyde dehydrogenase 1 (11), doublecortin-like kinase 1 (12), CD133 (13), c-Met (14) and CD44 mutant isoforms (CD44v) (15). Nevertheless, few studies have examined this population of cells, and it would be useful to identify new biomarkers for P-CSLCs. Our previous study developed a method for 
generating a P-CSLC-enriched population of cells with high CD44 and CD24 expression using pancreatic cancer cell lines (16), therefore this method maybe useful for identifying P-CSCL biomarkers.

Cathepsin B (CTSB) is a type of lysosomal cysteine protease $(17,18)$ that is synthesized as a 339 -amino acid preproenzyme with a 17 amino acid signal peptide on the rough endoplasmic reticulum and is associated with general protein turnover in lysosomes $(19,20)$. This protein is subject to multiple levels of regulation and may be involved in generally cancer progression (21). The SP1, SP3 and ETS1 proteins activate CTSB transcription (22), with SP1 and SP3 proteins being highly expressed generally in cancer cells and tissues (23), and ETS1 is associated with cancer invasion $(24,25)$. Therefore, the present study evaluated whether CTSB expression was upregulated in P-CSLCs and whether this was associated with patients' postoperative outcomes.

\section{Materials and methods}

Patients and tissue samples. The present study involved in vitro experiments using human pancreatic cancer cell lines, as well as a retrospective review of specimens from Japanese patients who underwent surgery for invasive ductal carcinoma. The patients were diagnosed according to the Japan Pancreas Society classification (26) and underwent radical resection with $\mathrm{D} 2$ or higher lymph node dissection between June 2001 and June 2013 at Yamaguchi University Hospital (Yamaguchi, Japan), and between March 2008 and October 2012 at Osaka University Hospital (Osaka, Japan). Written informed consent was obtained from participant at each institution. Treatment with gemcitabine alone, gemcitabine plus radiation, gemcitabine/S-1 plus radiation or gemcitabine plus heavy ion radiation was administered if patients received preoperative therapy. Treatment with gemcitabine alone, S-1 alone, gemcitabine plus immune cell therapy or immune cell therapy alone was administered if patients received postoperative therapy. Patients were excluded if they died from surgery-related causes or if they had other cancer types, serous and mucinous cystic pancreas neoplasms, pancreatic cancer derived from intraductal papillary-mucinous neoplasms, were pathologically cancer positive or had indeterminate surgical margins. Resected specimens without residual cancer were not considered. In total, 77 patients from Yamaguchi University Hospital and 64 patients from Osaka University Hospital were evaluated, although only 69 patients were considered eligible. The patients' medical records were reviewed to obtain their clinicopathological characteristics as listed in Table I, and the study protocol was approved by the Institutional Review Boards of Yamaguchi University Hospital (Yamaguchi, Japan) and Osaka University Hospital (Osaka, Japan). Tumor-Node-Metastasis (TNM) staging was performed according to the Union for International Cancer Control criteria (27).

Cell lines and culture conditions. The human pancreatic cancer cell line YPK2 has been established at Yamaguchi University School of Medicine (28). A common pancreatic cancer cell line, PANC-1, was purchased from the American Type Culture Collection. The cells were maintained at $37^{\circ} \mathrm{C}$ and 5\% $\mathrm{CO}_{2}$ in DMEM-F12 (Sigma-Aldrich; Merck KGaA) supplemented with $10 \%$ heat-inactivated fetal bovine serum (FBS) (Thermo Fisher Scientific, Inc.).

Generation of P-CSLCs. The P-CSLC-enriched populations were generated from YPK2 and PANC-1 cells as previously described (16). The cells were initially cultured in serum-free medium containing leukemia inhibitory factor (Merck KGaA), neural survival factor-1 (Lonza Group, Ltd.) and N-acetyl-L-cysteine (Sigma-Aldrich; Merck KGaA) to induce tumor sphere formation. The spheres were collected and transferred to laminin-coated dishes with culture medium containing B27 supplement (Thermo Fisher Scientific, Inc.), human recombinant epidermal growth factor (Sigma-Aldrich; Merck KGaA) and basic fibroblast growth factor (Merck KGaA). One-half of the culture medium was changed every week. The resultant cells were designated YPK2-Lm and PANC-1-Lm.

Two-dimensional (2D) electrophoresis and matrix-assisted laser desorption/ionization time of flight mass spectrometry and tandem mass spectrometry (MALDI TOF/TOF MS). Dead cells were eliminated from the cultures by labeling with Dead Cell Removal MicroBeads (Miltenyi Biotech GmbH) and separated using an LS column with a MidiMACS Separator (Miltenyi Biotech GmbH). CD44v9-positive cells were selected using rat anti-CD44v9 IgG (1:50; clone RV3; cat. no. LKG-M003; Cosmo Bio Co., Ltd.), mouse biotin-conjugated anti-rat IgG (1:2,000; cat. no. 13-4813-85; eBioscience; Thermo Fisher Scientific, Inc.) and microbeads carrying mouse anti-biotin IgG (ready to use; cat. no. 130-090-485; Miltenyi Biotech $\mathrm{GmbH}$ ), with isolation performed using the MidiMACS Separator according to the manufacturer's instructions.

Each sample was suspended in $0.2 \%$ pharmalyte and homogenized in lysis buffer containing $5 \mathrm{M}$ urea, $2 \mathrm{M}$ thiourea, $2 \%(\mathrm{w} / \mathrm{v})$ CHAPS, $2 \%(\mathrm{w} / \mathrm{v}) \mathrm{SB} 3-10$ and $1 \%(\mathrm{w} / \mathrm{v})$ DTT (all reagents from Sigma-Aldrich; Merck KGaA). Protein concentrations were measured using a protein assay kit (Bio-Rad Laboratories, Inc.). The samples were subjected to $2 \mathrm{D}$ electrophoresis as previously described $(29,30)$. Briefly, the samples were applied to 18-cm Immobiline DryStrips ( $\mathrm{pH} 3.0-10.0$; GE Healthcare) and then subjected to isoelectric focusing using CoolPhoreStar IPG-IEF Type-P (Anatech-Analytical Technology). The DryStrips were then subjected to 2D gradient electrophoresis (9-18\% acrylamide; FUJIFILM Wako Pure) using an ANDERSON ISO-DALT Multiple Electrophoresis system (Hoefer Inc.). After staining with SYPRO Ruby stain (S21900; Thermo Fisher Scientific, Inc.), protein spots were detected using a Molecular Imager FX (Bio-Rad Laboratories, Inc.) and analyzed using ImageMaster 2D Platinum software version 5.0 (GE Healthcare). Common protein spots with higher intensities in the YPK2-Lm cells (vs. the respective parental cells) were excised and subjected to MALDI TOF/TOF MS analysis.

The excised samples were de-stained, washed and dehydrated with acetonitrile. The gels were rehydrated in a digestion solution containing $50 \mathrm{mM} \mathrm{NH} \mathrm{NCO}_{3}, 5 \mathrm{mM} \mathrm{CaCl}_{2}$ and $0.01 \mu \mathrm{g} / \mu \mathrm{l}$ trypsin (Promega Corporation), and the digestion was then terminated using 5\% TFA. Peptides were extracted using 5\% TFA in 50\% acetonitrile. The samples were absorbed 
Table I. Association between 30 cases of low cathepsin B expression and 39 cases of high expression and clinical features of patients with pancreatic cancer.

\begin{tabular}{|c|c|c|c|}
\hline \multirow[b]{2}{*}{ Clinical feature } & \multicolumn{2}{|c|}{$\begin{array}{c}\text { Cathepsin B } \\
\text { expression }\end{array}$} & \multirow[b]{2}{*}{ P-value } \\
\hline & Low & High & \\
\hline Age, years & & & 0.366 \\
\hline$<60$ & 8 & 6 & \\
\hline$\geq 60$ & 22 & 33 & \\
\hline \multicolumn{4}{|l|}{ Sex } \\
\hline Male & 8 & 19 & 0.083 \\
\hline Female & 22 & 20 & \\
\hline Tumor location & & & 0.008 \\
\hline Pancreatic head & 15 & 32 & \\
\hline Pancreatic body and tail & 15 & 7 & \\
\hline Tumor size, mm & & & 0.045 \\
\hline$<30$ & 23 & 20 & \\
\hline$\geq 30$ & 28 & 35 & \\
\hline Differentiation & & & 0.690 \\
\hline Well & 2 & 4 & \\
\hline Moderate-poor & 28 & 35 & \\
\hline Invasion depth & & & 0.002 \\
\hline $\mathrm{T} 1+\mathrm{T} 2$ & 9 & 1 & \\
\hline $\mathrm{T} 3$ & 21 & 38 & \\
\hline Lymph node metastasis & & & 0.016 \\
\hline Negative & 19 & 13 & \\
\hline Positive & 11 & 26 & \\
\hline TNM stage & & & 0.038 \\
\hline $\mathrm{I}$ & 6 & 1 & \\
\hline II & 24 & 38 & \\
\hline Perineural invasion & & & 0.488 \\
\hline Negative & 5 & 4 & \\
\hline Positive & 25 & 35 & \\
\hline Portal invasion & & & 0.803 \\
\hline Negative & 19 & 26 & \\
\hline Positive & 11 & 13 & \\
\hline Preoperative therapy ${ }^{\dagger}$ & & & 0.058 \\
\hline None & 18 & 32 & \\
\hline Performed & 12 & 7 & \\
\hline Postoperative therapy & & & 0.532 \\
\hline None & 4 & 8 & \\
\hline Performed & 26 & 31 & \\
\hline
\end{tabular}

Data were analyzed using Fisher's exact test or $\chi^{2}$ where appropriate. 'Preoperative therapy included gemcitabine alone $(n=1)$, gemcitabine plus radiation $(n=10)$, gemcitabine/S-1 plus radiation $(n=7)$ and gemcitabine plus heavy ion radiation $(\mathrm{n}=1)$. ${ }^{*}$ Postoperative therapy included gemcitabine alone $(n=1), S-1$ alone $(n=34)$, gemcitabine plus immune cell therapy $(n=16)$ and immune cell therapy alone $(n=6)$. TNM, Tumor-Node-Metastasis.

to ZipTip $\mathrm{C} 18$ pipette tips (Merck $\mathrm{KGaA}$ ) and the peptides were eluted using $0.1 \%$ TFA in $50 \%$ acetonitrile. An aliquot of the eluted sample was mixed with an equal volume of matrix solution ( $0.3 \mathrm{~g} / \mathrm{l}$ alpha-cyano-4-hydroxycinnamic acid, 33\% acetone and $66 \%$ ethanol; all from Wako Chemicals $\mathrm{GmbH}$ ), placed onto a target plate (MTP Anchorchip 600/384; Bruker Corporation), dehydrated using MTP Anchorchip and analyzed using a mass spectrometer (Ultraflex TOF/TOF; Bruker Corporation) in the positive ion reflector mode $(20-4,000 \mathrm{~m} / \mathrm{z})$. The MS/MS spectra were searched against the NCBI NR database within the Mascot database search engine (Matrix Science, Ltd.).

Western blotting. Cells were lysed in a buffer containing $50 \mathrm{mM}$ Tris- $\mathrm{HCl}$ ( $\mathrm{pH} 8.0), 5$ mM EDTA, $5 \mathrm{mM}$ EGTA, $0.2 \%$ SDS, $0.5 \%$ Nonidet P-40, $1 \mathrm{mM} \mathrm{Na}_{3} \mathrm{VO}_{4}, 20 \mathrm{mM}$ sodium pyrophosphate and Roche complete protease inhibitor mixture. The protein amount was quantified by the Lowry method using DC Protein Assay (Bio-Rad Laboratories, Inc.). The proteins (10 $\mu \mathrm{g} /$ lane) were loaded onto a $8 \%$ gel, resolved using SDS-PAGE and transferred onto a PVDF membrane (Bio-Rad Laboratories, Inc.). Membranes were blocked with 3\% skimmed milk at room temperature for $1 \mathrm{~h}$ and treated with anti-CTSB (cat. no. ab58802; Abcam) and anti-valosin-containing protein (VCP; cat. no. GTX113030; GeneTex, Inc.) primary antibodies at room temperature for $1 \mathrm{~h}$, before the immunoreactive bands were visualized using an ECL Pro kit (PerkinElmer, Inc.) and Amersham Imager (GE Healthcare), with quantification using ImageJ software version 1.5.1 (National Institutes of Health). VCP was used as the loading control, because the protein levels of VCP is more stable compared with used loading controls such as GAPDH and actin (31-33). Moreover, the size of VCP $(97 \mathrm{kDa})$ is useful as a loading control for most of the proteins analyzed in this study were $<75 \mathrm{kDa}$.

Flow cytometry. Expression of CTSB on the surface of YPK2 and YPK2-Lm cells was analyzed using flow cytometry. Dissociated cells were suspended in PBS with $2 \%$ FBS $\left(10^{6}\right.$ cells $\left./ 100 \mu \mathrm{l}\right)$ and then incubated with each antibody at $4^{\circ} \mathrm{C}$ for $30 \mathrm{~min}$. The antibodies for this assay were rat anti-human CD44v9 (1:50; clone RV3; cat. no. LKG-M003; Cosmo Bio Co., Ltd.), anti-calreticulin (CALR; 1:50; cat. no. ab196159; Abcam) and anti-CTSB (1:20; cat. no. ab58802; Abcam). The mouse FITC-conjugated anti-rat IgG2a secondary antibody (1:10; cat. no. 11-4811-85; eBioscience; Thermo Fisher Scientific, Inc.) was used for the anti CD44v9 primary antibody. The anti-CTSB and cognate isotype control antibodies were conjugated with APC using an APC conjugation kit (cat. no. ab201807; Abcam) according to the manufacturer's instructions. Rat IgG2a $\kappa$ Isotype Control (cat. no. 14-4321-82; eBioscience; Thermo Fisher Scientific, Inc.), rabbit IgG Isotype Control Alexa Fluor 647 (cat. no. ab199093; Abcam) and mouse IgG2a $\kappa$ monoclonal Isotype Control (cat. no. ab18415; Abcam) were used as negative controls for anti-CD44v9, anti-CRT Alexa Fluor 647 and anti-CTSB with corresponding dilution factors. Flow cytometry analysis was performed using a FACS ARIA-III (BD Biosciences) and a MACSQuant analyzer version 2.4 (Miltenyi Biotec $\mathrm{GmbH}$ ).

ELISA. To quantify CTSB secretion, conditioned medium was collected and analyzed using a human cathepsin B ELISA kit (cat. no. ab119584; Abcam) according to the manufacturer's 
instructions. Signals were detected using an EnVision plate reader (PerkinElmer, Inc.).

Immunohistochemistry. Immunohistochemical staining for CTSB was performed using formalin-fixed paraffin-embedded surgical specimens (fixed in $10 \%$ formalin at room temperature overnight). Antigens in the specimens of 4- $\mu \mathrm{m}$ thickness were retrieved by heating the tissues for $20 \mathrm{~min}$ at $95^{\circ} \mathrm{C}$ in 10 mM Target Retrieval Solution, pH 6.0 (Dako; Agilent Technologies, Inc.) and then boiling for $10 \mathrm{~min}$ at $105^{\circ} \mathrm{C}$. Endogenous peroxidase activity was blocked using $3 \% \mathrm{H}_{2} \mathrm{O}_{2}$ in methanol for $5 \mathrm{~min}$ at room temperature, and non-specific binding was blocked using Protein Block serum-free (Dako; Agilent Technologies, Inc.) for $10 \mathrm{~min}$ at room temperature. The slides were incubated with the mouse monoclonal anti-CTSB antibodies (1:3,000; cat. no. ab58802; Abcam) for $1 \mathrm{~h}$ at room temperature and followed by the rat anti-mouse antibodies (undiluted solution; cat. no. K4001; Dako; Agilent Technologies, Inc.) for $30 \mathrm{~min}$ at room temperature. Tissue sections were incubated with 3,3'-diaminobenzidine tetrahydrochloride (Dako; Agilent Technologies, Inc.), and then counter-stained using hematoxylin for $2 \mathrm{~min}$ at room temperature.

Intensity of CTSB staining in the infiltrating pancreatic cancer specimen was classified as either negative or positive following examination of the positive area by a pathologist (AO) who was blinded to the patients' characteristics. High CTSB was defined as positive staining that occupied $>30 \%$ of $>5$ fields of view at 100 -fold magnification using a phase-contrast microscope (BZ-X700; KEYENCE). Low CTSB was defined as both negative and positive staining that occupied $<30 \%$ of the fields of view. Moderate staining was defined as a result between high and low staining based on the criteria using only occupation of positive cells as aforementioned. The brightness/contrast adjustment was applied to the whole image.

Statistical analysis. Each experiment was repeated at least three times. Data are expressed as mean \pm standard deviation. For the statistical analyses, patients with high and low CTSB expression were compared. The patients' clinicopathological characteristics and clinical outcomes were compared using the Fisher's exact test. Survival outcomes were analyzed using the Kaplan-Meier method and log-rank test. Significant differences between two or three groups were evaluated by Welch's t-test or ANOVA with Scheffe's test, respectively. All analyses were performed using $\mathrm{R}$ version 3.4.0 software (http://www.r-project.org/). $\mathrm{P}<0.05$ was considered to indicate a statistically significant difference.

\section{Results}

Identification and expression of CTSB. The protein expressions from YPK2-Lm and YPK5-Lm cells (generated P-CSLCs) and parental YPK2 and YPK5 cells were compared using proteome analysis using MALDI TOF/TOF MS data from previously performed 2D gel electrophoresis (30). The 2D gel electrophoresis revealed a 10-fold stronger band in YPK2-Lm compared with YPK2 cells (Fig. 1C and D), and the MALDI TOF/TOF MS assay identified CTSB (Fig. 1E).

\section{A (YPK2)}

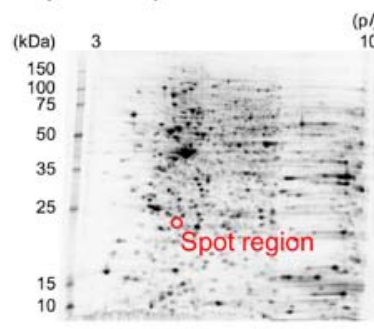

C (YPK2)

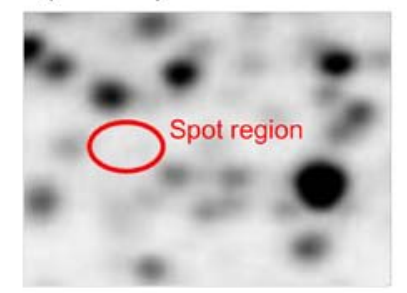

E

1 mwqlwaslcc llvlanarsr psfhplsdel vnyvnkrntt wqaghnfyn 1 dmsylkrlcg tflggpkppg rvmftedlkl pasfdareqw pqcptikei 101 dqgscgscwa fgaveaisdr icihtnahvs vevsaedllt ccgsmcgdgc 151 nggypaeawn fwtrkglvsg glyeshvgcr pysippcehh vngsrppctg 201 egdtpkcski cepgysptyk qdkhygynsy svsnsekdim aeiykngpve 251 gafsvysdfl lyksgvyghv tgemmgghai rilgwgveng tpywlvansw 301 ntdwgdngff kilrgqdhcg iesevvagip rtdqyweki

\section{$\mathrm{F}$}

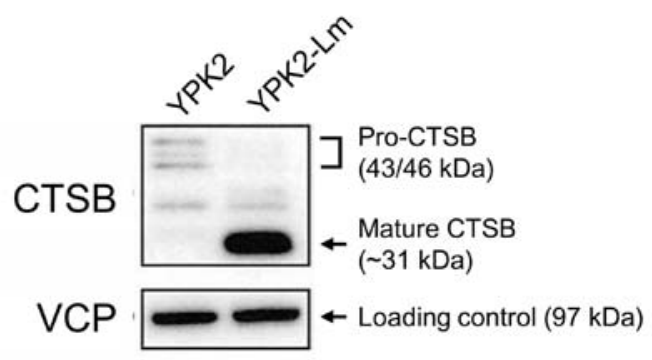

Figure 1. Identification of CTSB by proteome analysis. Representative two-dimensional gel electrophoresis images were previously shown (25). These images were obtained using proteins from (A and C) YPK2 and (B and D) YPK2-Lm. Red circles in C and D showed a 10-fold stronger corresponding spot in the YPK2-Lm compared with parental YPK2. (E) Identification of CTSB using matrix-assisted laser desorption/ionization time of flight mass spectrometry and tandem mass spectrometry, with matched peptides shown in red $(264-281$; score $=10,315-30$; score $=50)$. (F) Western blot analysis of YPK2 and YPK2-Lm. Anti-CTSB antibody employed in these analyses detects both mature CTSB $(\sim 31 \mathrm{kDa})$ and pro-CTSB (43/46 kDa). The expression level of VCP was used as a loading control. CTSB, cathepsin B; VCP, valosin-containing protein.

Western blotting confirmed that CTSB expression was elevated in YPK2-Lm cells compared with parental cells (Fig. 1F). Corresponding spots identified as CTSB were also obtained in the gels from YPK5 derivatives (data not shown). Protein expression on the surface of YPK2 and YPK2-Lm cells was evaluated using flow cytometry, and the results confirmed a slight elevated expression of CTSB on the surface of YPK2-Lm cells (Fig. 2). Slightly elevated expressions of CD44v9 and CALR were observed on the YPK2-Lm cell (Fig. 3) confirmed that P-CSLCs had been generated as described in a previous study (30). Elevated CTSB and CD44v9 expression was also seen in PANC-1-Lm cells compared with those in parental PANC-1 cells (Figs. S1 and S2). 


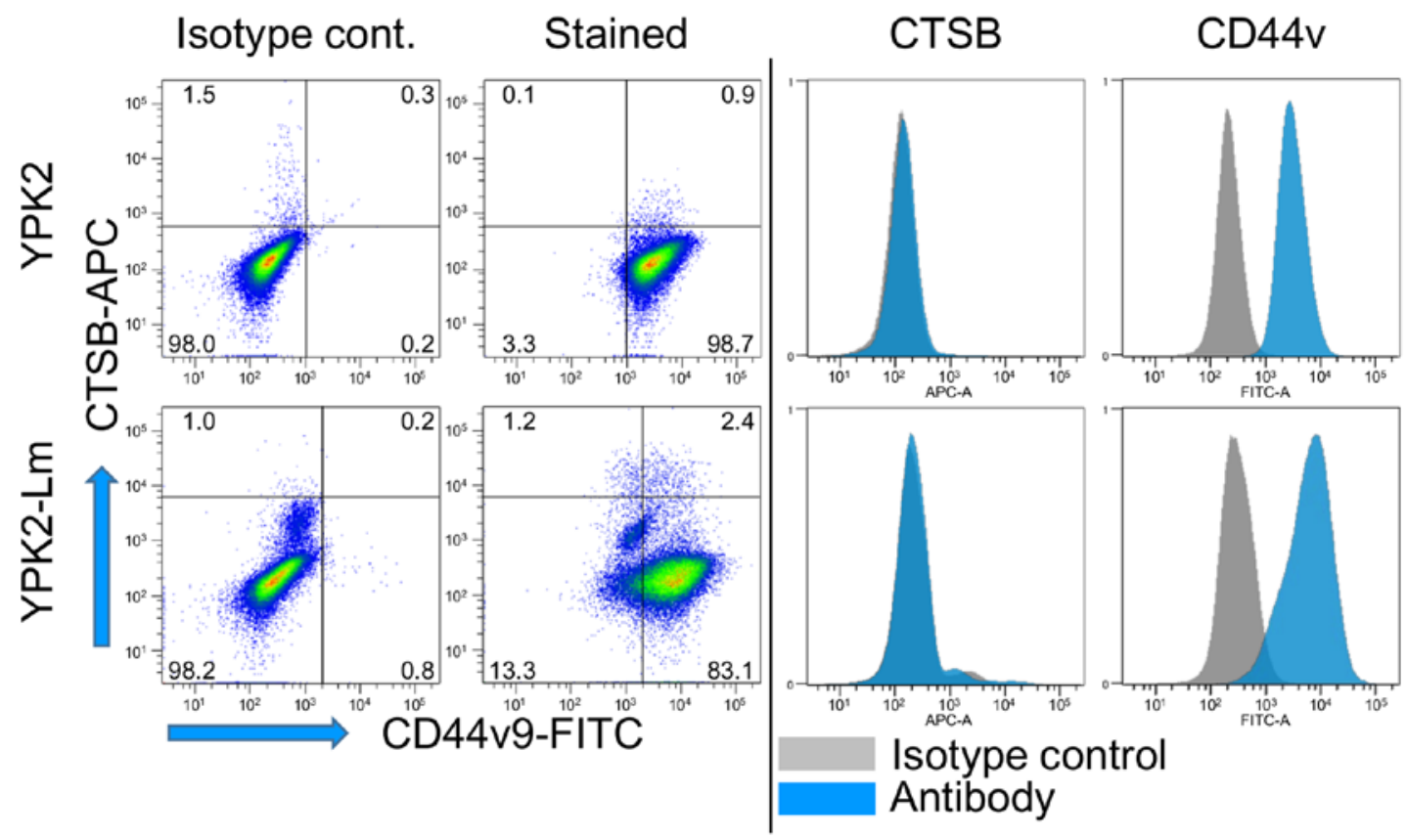

Figure 2. Expression of CTSB and CD44v9 on the surface of YPK2-Lm cells and YPK2 parental cells. Cells were stained with APC-conjugated anti-CTSB antibody and anti-CD44v9 antibody followed by FITC-conjugated anti-rat IgG2a secondary antibody and then separated using flow cytometry. Results indicated that CTSB was more highly expressed on the surface of YPK2-Lm cells compared with on YPK2 cells. Red histograms represent cells stained with isotype control antibodies. CTSB, cathepsin B; CD44v, variant isoforms of CD44.

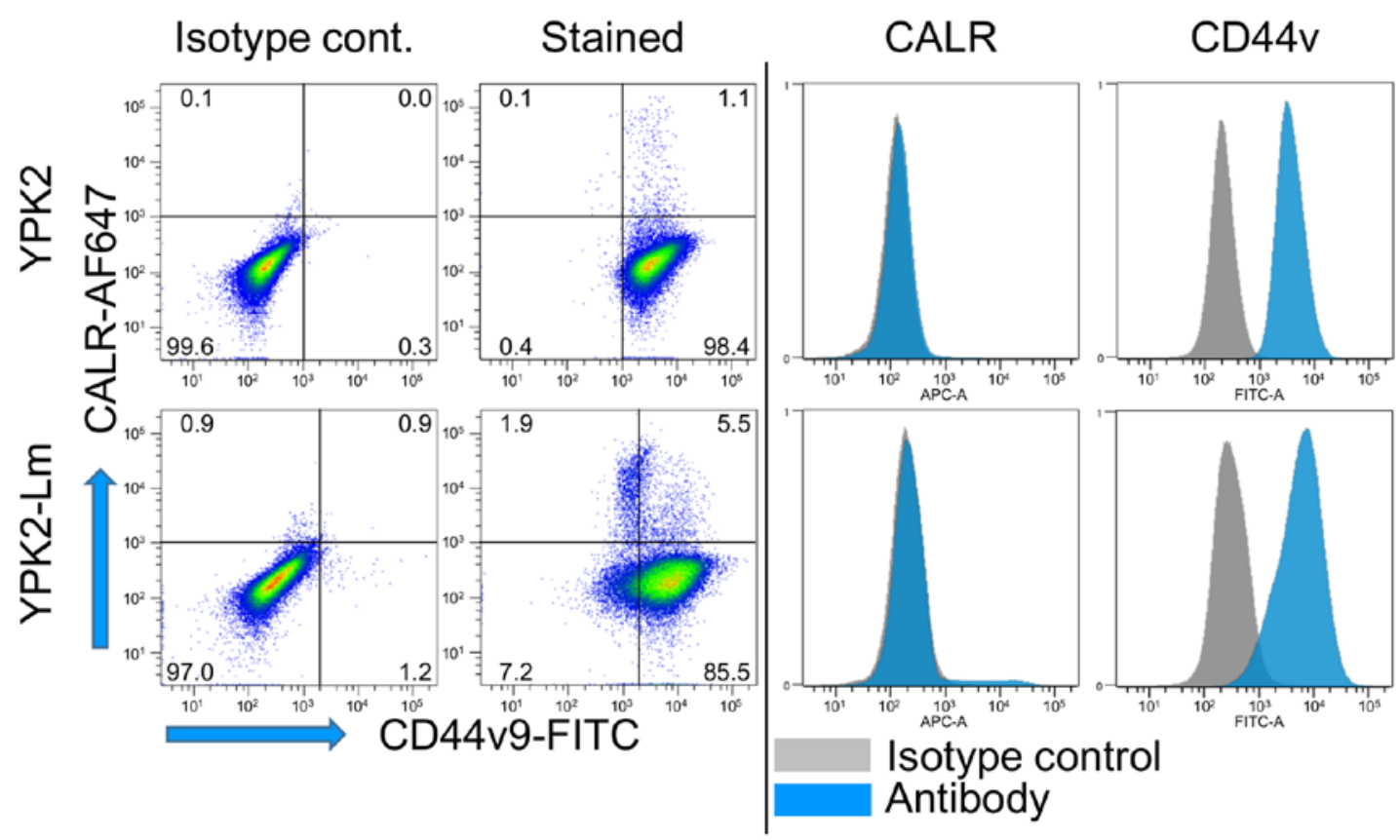

Figure 3. Expression of calreticulin and CD44v9 on the surface of YPK2-Lm cells and YPK2 parental cells. Cells were stained with Alexa Fluor 647-conjugated anti-CALR antibody and anti-CD44v9 antibody followed by FITC-conjugated anti-rat IgG2a secondary antibody and then separated by flow cytometry. Results indicated that CALR and CD44v9 were more highly expressed on the surface of YPK2-Lm cells compared with on YPK2 cells. Red histograms represent cells stained with isotype control antibodies. CD44v, variant isoforms of CD44; CALR, calreticulin; cont., control.

Association between CTSB expression and clinical outcomes. The patient selection process is shown in Fig. 4A, and representative images of CTSB expression in the tumor specimens are shown in Fig. 4B. While normal tissues exhibited minimal CTSB staining, CTSB expression was predominantly detected in the cytoplasm of cancerous tissues, although some cases also exhibited nucleus or membranous staining (Fig. 4B). In addition to those staining location, there was broad variation, ranging from diffuse staining to staining involving granulocytes or even the Golgi area. Comparison of the clinical outcomes between the groups with low and high CTSB expression revealed that high expression was significantly associated with more advanced disease parameters, including tumor size $(\mathrm{P}=0.045)$, invasion depth $(\mathrm{P}=0.002)$, lymph node 


\section{A}

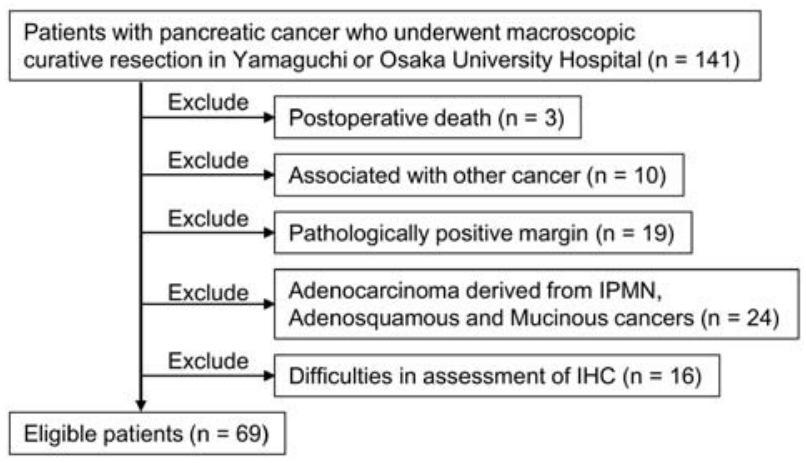

B
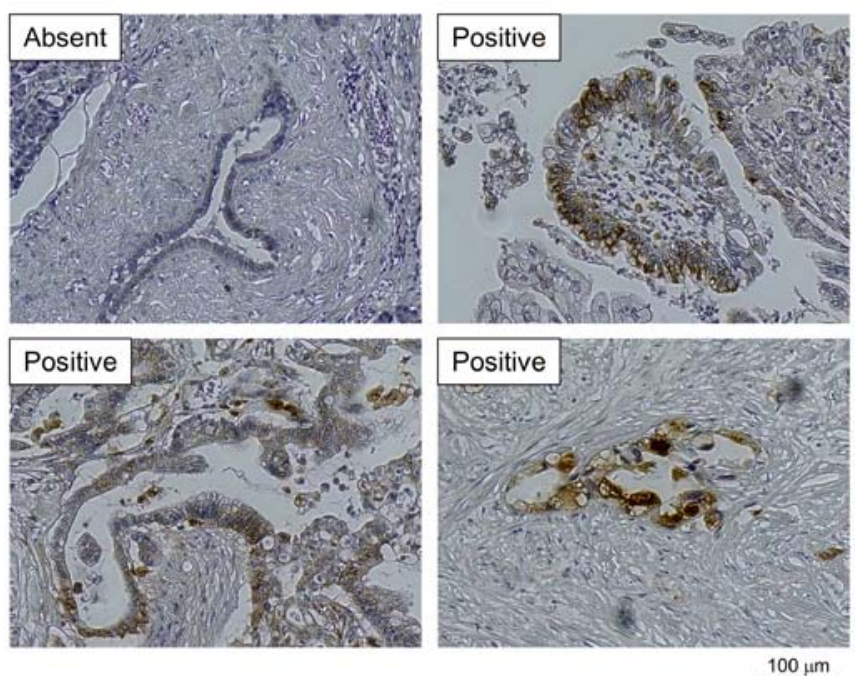

$100 \mu \mathrm{m}$

Figure 4. CTSB expression in resected pancreatic tumor samples. (A) Study flowchart. (B) CTSB expression in resected pancreatic tumor samples, categorized as negative (upper left panel) or positive (lower and upper right panels). CTSB, cathepsin B; IPMN, intraductal papillary mucinous neoplasm; IHC, immunohistochemistry.

metastasis $(\mathrm{P}=0.016)$ and TNM stage $(\mathrm{P}=0.038)$ (Table $\mathrm{I})$. Furthermore, the high expression group had significantly more cases with pancreatic head tumors, as well as a lower overall survival rate, compared with those with low CTSB expression (Fig. 5).

Secretion of CTSB into YPK2 growth medium. ELISA was used to evaluate the cellular secretions of CTSB by YPK2 and YPK2-Lm cells. The results revealed significantly elevated concentrations of CTSB in the medium for YPK2-Lm cells compared with YPK2 cells ( $<<0.05$; Fig. 6). The same result was obtained with for PANC-1-Lm compared with PANC-1 cells ( $\mathrm{P}<0.05$; Fig. S3).

\section{Discussion}

The function of CTSB is dynamic and poorly understood; however, it is hypothesized that CTSB encoding lysosomal proteinase plays a role in not only in normal physiological conditions but also in pathological conditions, such as inflammation, infection and neurodegenerative disease, and the malignant progression of several cancer types including

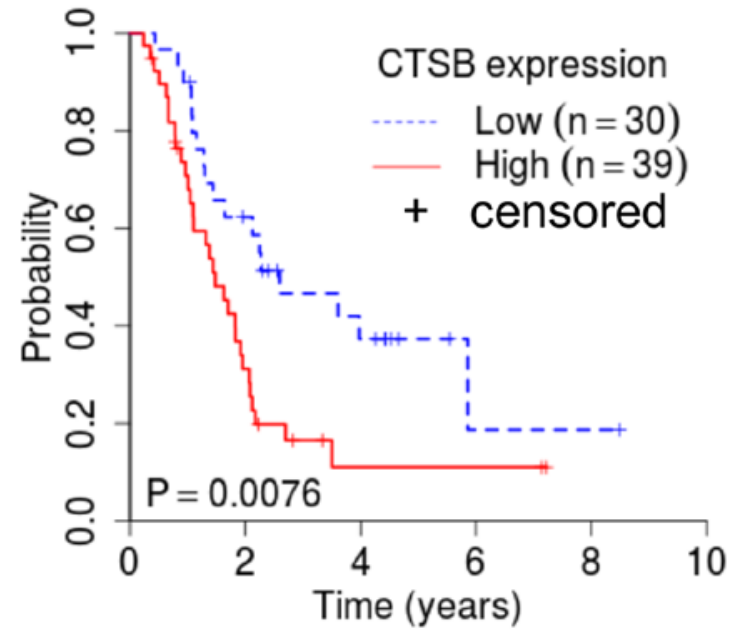

Figure 5. A Kaplan-Meier curve for CTSB expression in patients with pancreatic cancer. Overall survival rate according to high CTSB expression (red line, 39 patients) or low expression (blue dotted line, 30 patients). A $\mathrm{P}$-value was calculated using the log-rank test. CTSB, cathepsin B.

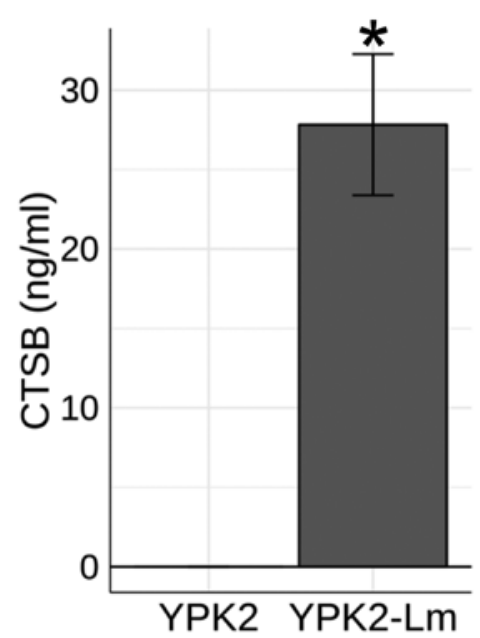

Figure 6. Concentrations of CTSB based on ELISA using media from cultures of YPK2 and YPK2-Lm cells. Results indicated that CTSB was present at a higher concentration in the culture media from YPK2-Lm cells compared with that from YPK2 cells. Each medium incubated in the absence of cells was used as a blank. Data were analyzed using Welch's t-test. ${ }^{*} \mathrm{P}<0.05$. CTSB, cathepsin B.

gastric, colorectal, breast and pancreatic cancers (34-38). It remains unclear whether CTSB is expressed in tumor cells, tumor-related cells, or both, and whether CTSB expression affects or is affected by interactions with cellular and/or non-cellular microenvironments (21). A previous study have previously described an association between CSC and CTSB expression (39).

The present study generated P-CSLCs using our previously reported method $(16,30)$, in which YPK2-Lm cells showed not only a much higher population of CD44v compared with parental YPK2 cells, but also much higher side-population and higher ALDH activity. In addition, the enhancement of tumorigenicity and epithelial-mesenchymal transition was confirmed by both mouse subcutaneous injection and quantitative PCR analyses. Protein analyses revealed that CTSB was more highly expressed in P-CSLCs compared with in the 
parent pancreatic cancer cell lines. Western blot analysis also confirmed that CTSB was highly expressed in the P-CSLCs, while flow cytometry revealed elevated CTSB expression on the surface of P-CSLCs. CD24, CD44, CD44v and CALR markers expressed on the cell surface are useful for the identification of pancreatic cancer stem cells (30). The present results suggested that CTSB cell surface expression and total protein levels could be used as prognostic biomarkers. The current study examined tumor specimens with high CTSB staining intensity, and observed that most of these cases exhibited strong cytoplasmic staining, although some cases also exhibited nucleus or membranous staining. There was broad variation in the staining location for these combined cases, ranging from diffuse staining to staining involving granulocytes or even the Golgi area. Moreover, high CTSB expression in resected specimens was significantly associated with advanced disease parameters (larger tumor size, deeper invasion depth, positive for lymph node metastasis and higher TNM stage) and lower overall survival rate, which is consistent with previously reported results (40). These results support the hypothesis that CTSB is associated with pancreatic cancer progression. It has been reported that CTSB contributes generally to tumor growth, migration, invasion and angiogenesis (38). Notably, the observation of both expressions of CTSB and its inhibitors in tumor tissues and tumor-infiltrating immune cells had suggested that those may be involved in pancreatic ductal adenocarcinoma-related inflammation (41).

Given the diffuse CTSB staining observed in the pancreatic cancer tissues as aforementioned, the secretion of CTSB was examined in vitro. Higher CTSB concentrations in the culture medium for P-CSLCs compared with the medium for the parental cells was observed, which suggested that CTSB is expressed on the cell surface of P-CSLCs and is also secreted by these cells. Thus, CTSB in P-CSLCs may directly or indirectly influence the extracellular microenvironment and potentially contribute to the progression of malignancy. These results were further confirmed using $\mathrm{P}-\mathrm{CSLCs}$ generated from two pancreatic cancer cell lines (PANC-1 and YPK2), which suggests that the present findings were not cell line-specific.

It should be highlighted that the present results do not clarify the functional role of CTSB in P-CSLCs, although CTSB in various locations (surface, intracellular and secretions) has been widely reported to be involved in the progression of various cancer types (40-49) including glioblastoma, malignant meningioma, pancreatic, breast, esophageal and prostate cancers. Notably, the subcellular localization of various cancer markers is generally limited to a single compartment, although their localization varies according to malignancy in some high-grade carcinomas. For example, cytokeratin is a common marker for solid tumor cells and is often expressed in the cell membrane in well-differentiated adenocarcinoma, but is expressed in the membrane and Golgi in high-grade poorly differentiated adenocarcinoma (50). Thus, multiple localizations for cancer-associated proteins may indicate a high-grade tumor. Similar to cytokeratin (50), CTSB is also a cancer-associated protein with multiple cellular localizations as shown in the present study, and the association between its localization and malignancy is not clear, although the present findings suggested that CTSB in cancer stem cells may be involved in the degree of malignancy.
The present study revealed that CTSB expression was elevated in P-CSLCs generated from common pancreatic cancer cell lines, and that its expression in resected tumor specimens was associated with poor postoperative outcomes. While it is unclear whether this protein is a useful therapeutic target, it might be a useful prognostic marker that is associated with pancreatic cancer stem cells, especially if it is present in multiple subcellular locations. Further studies with gene-targeting experiments such as genome-editing and in vivo murine experiments are needed to clarify the prognostic implications of CTSB expression in this setting, as well as whether it is a potential therapeutic target.

\section{Acknowledgements}

The authors would like to thank Ms. Akiko Sano, Ms. Kaori Kaneyasu and Ms. Hiroko Takenouchi for their technical assistance (Department of Gastroenterological, Breast and Endocrine Surgery, Yamaguchi University Graduate School of Medicine).

\section{Funding}

The study was funded by JSPS KAKENHI Grants (grant nos. 24390317, 17H06903, 18K16365, and 18K08646).

\section{Availability of data and materials}

The datasets used and/or analyzed during the present study are available from the corresponding author on reasonable request.

\section{Authors' contributions}

TF, SM, KY and HN conceived and designed the present study. TF, SM, AO, NF and YF performed the experiments. Statistical analysis was performed by TF and RT. TF, RT and HN wrote the manuscript. RT, KY, HM, YS, YT, NS, SK, SH and HE interpreted the data and edited the manuscript. All authors read and approved the final manuscript.

\section{Ethics approval and consent to participate}

The study protocol was approved by the Institutional Review Boards of Yamaguchi University Hospital (protocol number, H27-007) and Osaka University Hospital (protocol number, 15208). All patients provided written informed consent.

\section{Patient consent for publication}

Not applicable.

\section{Competing interests}

The authors declare that they have no competing interests.

\section{References}

1. The Editorial Board of the Cancer Statistics in Japan: Cancer statistics in Japan 2018. Foundation for Promotion of Cancer Research, Tokyo, pp 15, 2019.

2. Ryan DP, Hong TS and Bardeesy N: Pancreatic adenocarcinoma. N Engl J Med 371: 1039-1049, 2014. 
3. Murakami T, Hiroshima Y, Matsuyama R, Homma Y, Hoffman RM and Endo I: Role of the tumor microenvironment in pancreatic cancer. Ann Gastroenterol Surg 3: 130-137, 2019.

4. Oettle H, Neuhaus P, Hochhaus A, Hartmann JT, Gellert K, Ridwelski K, Niedergethmann M, Zülke C, Fahlke J, Arning MB, et al: Adjuvant chemotherapy with gemcitabine and long-term outcomes among patients with resected pancreatic cancer: The CONKO-001 randomized trial. JAMA 310: 1473-1481, 2013.

5. Torphy RJ, Zhu Y and Schulick RD: Immunotherapy for pancreatic cancer: Barriers and breakthroughs. Ann Gastroenterol Surg 2: 274-281, 2018.

6. Clevers H: The cancer stem cell: Premises, promises, and challenges. Nat Med 17: 313-319, 2011.

7. Li X, Lewis MT, Huang J, Gutierrez C, Osborne CK, Wu MF Hilsenbeck SG, Pavlick A, Zhang X, Chamness GC, et al: Intrinsic resistance of tumorigenic breast cancer cells to chemotherapy. J Natl Cancer Inst 100: 672-679, 2008.

8. Zhou BB, Zhang H, Damelin M, Geles KG, Grindley JC and Dirks PB: Tumour-initiating cells: Challenges and opportunities for anticancer drug discovery. Nat Rev Drug Discov 8: 806-823, 2009.

9. Tsunedomi R, Yoshimura K, Suzuki N, Hazama S and Nagano H: Clinical implications of cancer stem cells in digestive cancers: Acquisition of stemness and prognostic impact. Surg Today: Feb 5, 2020 (Epub ahead of print). doi: 10.1007/s00595-020-01968-x.

10. Li C, Heidt DG, Dalerba P, Burant CF, Zhang L, Adsay V, Wicha M, Clarke MF and Simeone DM: Identification of pancreatic cancer stem cells. Cancer Res 67: 1030-1037, 2007.

11. Rasheed ZA, Yang J, Wang Q, Kowalski J, Freed I, Murter C, Hong SM, Koorstra JB, Rajeshkumar NV, He X, et al: Prognostic significance of tumorigenic cells with mesenchymal features in pancreatic adenocarcinoma. J Natl Cancer Inst 102: 340-351, 2010.

12. Sureban SM, May R, Qu D, Weygant N, Chandrakesan P, Ali N, Lightfoot SA, Pantazis P, Rao CV, Postier RG and Houchen CW: DCLK1 regulates pluripotency and angiogenic factors via microRNA-dependent mechanisms in pancreatic cancer. PLoS One 8: e73940, 2013.

13. Hermann PC, Huber SL, Herrler T, Aicher A, Ellwart JW, Guba M, Bruns CJ and Heeschen C: Distinct populations of cancer stem cells determine tumor growth and metastatic activity in human pancreatic cancer. Cell Stem Cell 1: 313-323, 2007.

14. Li C, Wu JJ, Hynes M, Dosch J, Sarkar B, Welling TH, Pasca di Magliano M and Simeone DM: c-Met is a marker of pancreatic cancer stem cells and therapeutic target. Gastroenterology 141: 2218-2227, 2011.

15. Ishimoto T, Nagano O, Yae T, Tamada M, Motohara T, Oshima $\mathrm{H}$, Oshima M, Ikeda T, Asaba R, Yagi H, et al: CD44 variant regulates redox status in cancer cells by stabilizing the $\mathrm{xCT}$ subunit of system xc (-) and thereby promotes tumor growth. Cancer Cell 19: 387-400, 2011.

16. Watanabe Y, Yoshimura K, Yoshikawa K, Tsunedomi R, Shindo Y, Matsukuma S, Maeda N, Kanekiyo S, Suzuki N, Kuramasu A, et al: A stem cell medium containing neural stimulating factor induces a pancreatic cancer stem-like cell-enriched population. Int J Oncol 45: 1857-1866, 2014.

17. Rawlings ND, Barrett AJ and Bateman A: MEROPS: The database of proteolytic enzymes, their substrates and inhibitors. Nucleic Acids Res 40: D343-D350, 2012.

18. Turk V, Stoka V, Vasiljeva O, Renko M, Sun T, Turk B and Turk D Cysteine cathepsins: From structure, function and regulation to new frontiers. Biochim Biophys Acta 1824: 68-88, 2012.

19. Kirschke H, Barrett AJ and Rawlings ND: Proteinases 1: Lysosomal cysteine proteinases. Protein Profile 2: 1581-1643, 1995

20. Mort JS and Buttle DJ: Cathepsin B. Int J Biochem Cell Biol 29: 715-720, 1997.

21. Aggarwal N and Sloane BF: Cathepsin B: Multiple roles in cancer. Proteomics Clin Appl 8: 427-437, 2014.

22. Yan S, Berquin IM, Troen BR and Sloane BF: Transcription of human cathepsin B is mediated by Sp1 and Ets family factors in glioma. DNA Cell Biol 79: 79-91, 2000.

23. Li L and Davie JR: The role of Sp1 and $\mathrm{Sp} 3$ in normal and cancer cell biology. Ann Anat 192: 275-283, 2010.

24. Dittmer J: The biology of the Ets1 proto-oncogene. Mol Cancer 2: 29, 2003.

25. Buggy Y, Maguire TM, McGreal G, McDermott E, Hill ADK, O'Higgins $N$ and Duffy MJ: Overexpression of the Ets-1 transcription factor in human breast cancer. $\mathrm{Br} \mathrm{J}$ Cancer 91: 1308-1315, 2004.
26. Japan Pancreas Society: General rules for the study of pancreatic cancer, The 6th Edition, Revised Version edn. Tokyo, Japan: Kanehara, pp 3-14, 2013.

27. Union for International Cancer Control (UICC): TNM Classification of Malignant Tumours, 7th edition. Sobin LH, Gospodarowicz MK and Wittekind C (eds.). Wiley, New York, NY, 2010 .

28. Yamamoto K, Yahara N, Gondo T, Ishihara T and Oka M: Establishment and characterization of a new human pancreatic cancer cell line, YPK-1. Bull Yamaguchi Med Sch 49: 33-42, 2002.

29. Gerashchenko BI, Yamagata A, Oofusa K, Yoshizato K, de Toledo SM and Howell RW: Proteome analysis of proliferative response of bystander cells adjacent to cells exposed to ionizing radiation. Proteomics 7: 2000-2008, 2007.

30. Matsukuma S, Yoshimura K, Ueno T, Oga A, Inoue $M$, Watanabe Y, Kuramasu A, Fuse M, Tsunedomi R, Nagaoka S, et al: Calreticulin is highly expressed in pancreatic cancer stem-like cells. Cancer Sci 107: 1599-1609, 2016.

31. Fujiwara N, Usui T, Ohama T and Sato K: Regulation of beclin 1 protein phosphorylation and autophagy by protein phosphatase 2A (PP2A) and death-associated protein kinase 3 (DAPK3). J Biol Chem 291: 10858-10866, 2016.

32. Enjoji S, Yabe R, Tsuji S, Yoshimura K, Kawasaki H, Sakurai M, Sakai Y, Takenouchi H, Yoshino S, Hazama S, et al: Stemness is enhanced in gastric cancer by a SET/PP2A/E2F1 axis. Mol Cancer Res 16: 554-563, 2008.

33. Yabe R, Tsuji S, Mochida S, Ikehara T, Usui T, Ohama T and Sato K: A stable association with PME-1 may be dispensable for PP2A demethylation-implications for the detection of PP2A methylation and immunoprecipitation. FEBS Open Bio 8: 1486-1496, 2018.

34. Ebert MP, Krüger S, Fogeron ML, Lamer S, Chen J, Pross M, Schulz HU, Lage H, Heim S, Roessner A, et al: Overexpression of cathepsin B in gastric cancer identified by proteome analysis. Proteomics 5: 1693-1704, 2005.

35. McKerrow JH, Bhargava V, Hansell E, Huling S, Kuwahara T, Matley M, Coussens L and Warren R: A functional proteomics screen of proteases in colorectal carcinoma. Mol Med 6: 450-460, 2000.

36. Srisomsap C, Subhasitanont P, Otto A, Mueller EC, Punyarit P, Wittmann-Liebold B and Svasti J: Detection of cathepsin B up-regulation in neoplastic thyroid tissues by proteomic analysis. Proteomics 2: 706-712, 2002.

37. Wulfkuhle JD, Sgroi DC, Krutzsch H, McLean K, McGarvey K, Knowlton M, Chen S, Shu H, Sahin A, Kurek R, et al: Proteomics of human breast ductal carcinoma in situ. Cancer Res 62: 6740-6749, 2002

38. Mijanović O, Branković A, Panin AN, Savchuk S, Timashev P, Ulasov I and Lesniak MS: Cathepsin B: A sellsword of cancer progression. Cancer Lett 449: 207-214, 2019.

39. Tang KD, Liu J, Jovanovic L, An J, Hill MM, Vela I, Lee TK, Ma S, Nelson C, Russell PJ, et al: Adipocytes promote prostate cancer stem cell self-renewal through amplification of the cholecystokinin autocrine loop. Oncotarget 7: 4939-4948, 2016.

40. Niedergethmann M, Wostbrock B, Sturm JW, Willeke F, Post S and Hildenbrand R: Prognostic impact of cysteine proteases cathepsin B and cathepsin $\mathrm{L}$ in pancreatic adenocarcinoma. Pancreas 29: 204-211, 2004.

41. Komura T, Takabatake H, Harada K, Yamato M, Miyazawa M, Yoshida K, Honda M, Wada T, Kitagawa H, Ohta T, et al: Clinical features of cystatin A expression in patients with pancreatic ductal adenocarcinoma. Cancer Sci 108: 2122-2129, 2017.

42. Krueger S, Haeckel C, Buehling $\mathrm{F}$ and Roessner A: Inhibitory effects of antisense cathepsin B cDNA transfection on invasion and motility in a human osteosarcoma cell line. Cancer Res 59: 6010-6014, 1999.

43. Shimizu A, Nakayama $\mathrm{H}$, Wang $\mathrm{P}$, König C, Akino $\mathrm{T}$, Sandlund J, Coma S, Italiano JE Jr, Mammoto A, Bielenberg DR and Klagsbrun M: Netrin-1 promotes glioblastoma cell invasiveness and angiogenesis by multiple pathways including activation of RhoA, cathepsin B, and cAMP-response element-binding protein. J Biol Chem 288: 2210-2222, 2013.

44. Withana NP, Blum G, Sameni M, Slaney C, Anbalagan A, Olive MB, Bidwell BN, Edgington L, Wang L, Moin K, et al: Cathepsin B inhibition limits bone metastasis in breast cancer. Cancer Res 72: 1199-1209, 2012. 
45. Tummalapalli P, Spomar D, Gondi CS, Olivero WC, Gujrati M, Dinh DH and Rao JS: RNAi-mediated abrogation of cathepsin B and MMP-9 gene expression in a malignant meningioma cell line leads to decreased tumor growth, invasion and angiogenesis. Int J Oncol 31: 1039-1050, 2007

46. Gocheva V, Zeng W, Ke D, Klimstra D, Reinheckel T, Peters C, Hanahan D and Joyce JA: Distinct roles for cysteine cathepsin genes in multistage tumorigenesis. Genes Dev 20: 543-556, 2006.

47. Andl CD, McCowan KM, Allison GL and Rustgi AK: Cathepsin B is the driving force of esophageal cell invasion in a fibroblast-dependent manner. Neoplasia 12: 485-498, 2010

48. Guo M, Mathieu PA, Linebaugh B, Sloane BF and Reiners JJ Jr: Phorbol ester activation of a proteolytic cascade capable of activating latent transforming growth factor-betaL a process initiated by the exocytosis of cathepsin B. J Biol Chem 277: 14829-14837, 2002.
49. Steffan JJ, Snider JL, Skalli O, Welbourne T and Cardelli JA: $\mathrm{Na}^{+} / \mathrm{H}^{+}$exchangers and RhoA regulate acidic extracellular $\mathrm{pH}$-induced lysosome trafficking in prostate cancer cells. Traffic 10: 737-753, 2009.

50. Bayrak R, Yenidünya S and Haltas H: Cytokeratin 7 and cytokeratin 20 expression in colorectal adenocarcinoma. Pathol Res Pract 207: 156-60, 2011.

(c) (7) (5) This work is licensed under a Creative Commons Attribution 4.0 International (CC BY-NC 4.0) License 